OPEN ACCESS

Edited by:

Wenting Liao,

Sun Yat-sen University Cancer Center

(SYSUCC), China

Reviewed by:

Justin Lathia,

Case Western Reserve University,

United States

Marc Mesnil,

University of Poitiers, France

*Correspondence:

Kai-Jun Luo

kaijun_luo@ynu.edu.cn

Yun-Chao Huang

huangych2001@aliyun.com

Jean X. Jiang

jiangj@uthscsa.edu

Specialty section:

This article was submitted to Molecular and Cellular Oncology,

a section of the journal

Frontiers in Oncology

Received: 27 August 2020

Accepted: 11 November 2020

Published: 23 December 2020

Citation:

Luo K-J, Chen C-X, Yang J-P,

Huang $Y-C$, Cardenas $E R$ and Jiang JX (2020) Connexins in Lung

Cancer and Brain Metastasis.

Front. Oncol. 10:599383.

doi: 10.3389/fonc. 2020.599383

\section{Connexins in Lung Cancer and Brain Metastasis}

\author{
Kai-Jun Luo ${ }^{1,2 *}$, Chang-Xu Chen ${ }^{1,2}$, Jia-Peng Yang ${ }^{3,4}$, Yun-Chao Huang ${ }^{3,4 *}$, \\ Eduardo R. Cardenas ${ }^{5}$ and Jean X. Jiang ${ }^{5 *}$
}

1 School of Life Sciences, Yunnan University, Kunming, China, ${ }^{2}$ Key Laboratory of the University in Yunnan Province for International Cooperation in Intercellular Communications and Regulations, Yunnan University, Kunming, China, ${ }^{3}$ Department of Thoracic Surgery I, The Third Affiliated Hospital of Kunming Medical University/Yunnan Cancer Hospital, Yunnan Cancer Center, Kunming, China, ${ }^{4}$ Joint International Research Laboratory of Regional Tumor in High Altitude Area, Kunming, China, ${ }^{5}$ Department of Biochemistry and Structural Biology, University of Texas Health Science Center, San Antonio, TX, United States

Connexins (Cxs) are involved in the brain metastasis of lung cancer cells. Thus, it is necessary to determine whether gap junction-forming Cxs are involved in the communication between lung cancer cells and the host cells, such as endothelial cells, forming the brain-blood-barrier, and cells in the central nervous system. Data from multiple studies support that Cxs function as tumor suppressors during lung cancer occurrence. However, recent evidence suggests that during metastasis to the brain, cancer cells establish communication with the host. This review discusses junctional or non-junctional hemichannel studies in lung cancer development and brain metastasis, highlighting important unanswered questions and controversies.

Keywords: lung cancer brain metastasis, connexin, gap junction, hemichannel, endothelial cells, astrocytes

\section{INTRODUCTION}

The understanding of Connexin $(\mathrm{Cx})$ channels in lung cancer brain metastasis is rather limited. In the human genome, $21 \mathrm{Cxs}$ are found (Figure 1) and in the mouse genome, $20 \mathrm{mCx}$ genes are found (3-6). A connexin hemichannel is also known as a connexon, and comprises a hemichannel assembly with six connexin subunits formed on the cell membrane; two hemichannels from neighboring cells dock together to form gap junctions $(\mathrm{GJs})(7,8)$. GJs are intercellular communication junctions, important in the maintenance of homeostasis, and their disruption is associated with pathology, particularly in carcinogenic processes $(9,10)$. GJs are responsible for intracellular communication via the passing of small ions and hydrophilic metabolites less than 1 $\mathrm{kDa}$ in size. In addition to forming gap junctions, hemichannels provide unique cell permeability between the intracellular and extracellular milieus in tissue function and affect tumorigenesis, suppression of cancer growth, and metastasis $(11,12)$. 


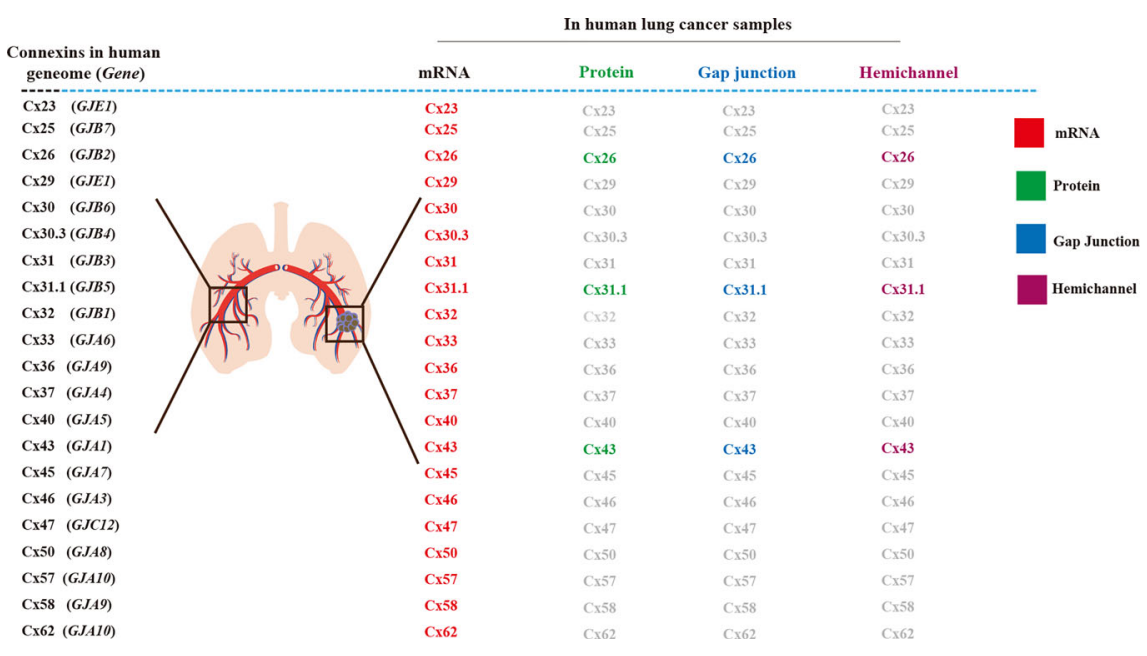

FIGURE 1 Connexins (Cxs) in human lung cancer tissue and its Cx-channels. In normal lung tissues, 21 connexin genes, mRNA, and protein have been detected in the human genome, junctional hemichannel, and hemichannels (1, 2).

Metastasis results in the dissemination of cancer cells to a new organ. Over half of the brain metastases are derived from lung adenocarcinoma. The dissemination of lung cancer cells, especially to brain parenchyma and leptomeninges, causes high morbidity; as this metastasis is primarily associated with the central nervous system, it leads to a poor prognosis (13). Lung cancer cells need to overcome several stages or barriers to metastasize to the brain. First, the cells must grow at their site of origin and detach from neighboring cells in the lung. Next, they must intravasate to nearby blood vessels, penetrating blood vessels, then cross the blood-brain barrier (diapedesis). Finally, to establish itself as a metastatic cancer cell in the brain, the cancer cells must undergo angiogenesis to form a new blood supply (14). However, the understanding of the role of $\mathrm{Cx}^{-}$ channels in lung cancer brain metastasis is limited, and necessitates further research.

\section{CONNEXINS, GAP JUNCTIONS, HEMICHANNEL, AND LUNG CANCER TYPES}

Different isoforms of $\mathrm{Cx}$ may have distinct functions in lung cancer tissues and cell lines.

Non-small cell lung cancer (NSCLC) and small cell lung cancer (SCLC) are the two foremost lung cancers; furthermore, NSCLC has been classified into three subclasses: NSCLC-la (large cell carcinoma), NSCLC-sq (squamous cell carcinoma), and NSCLC-ad (adenocarcinoma). These subclasses normally stem from the bronchioles, small alveoli, and alveoli (15).

Cx expression at the transcriptional and protein translation levels have been observed in human lung cancer tissues, using electrophysiology and dye uptake techniques, to identify $\mathrm{Cx}$ biomarkers for clinical prognosis. To date, $21 \mathrm{Cx}$ mRNAs have been detected in lung cancer tissues including in NSCLC-ad and NSCLC-sq, with differential abundance compared to that in normal healthy lung tissues (1). Although the mRNA levels of these Cxs do not represent hemichannel functionality or gap junction intercellular communication (GJIC), researchers have demonstrated that high $\mathrm{Cx}$ mRNA levels are associated with better survival, suggesting the association of $\mathrm{Cx}$ expression with tumor-suppressive roles (1). Other indicators suggest that Cxs may also function as tumor promoters, as their expression correlates with poor survival (1). Therefore, information such as cancer stage and cancer type is key (1). Cx26 significantly correlates with poor prognosis and metastasis in NSCLC-sq (2). The protein, hemichannel, and GJ functions of Cxs, such as $\mathrm{Cx} 43$ (1) and $\mathrm{Cx} 26$ require further investigation (2) (Figure 1).

An interesting link between Cxs and lung cancer brain metastasis has been observed in the NSCLC-la cell line SKLuCi-6, derived from lung cancer brain metastatic tumors, which present detectable levels of GJIC. However, other SCLC cell lines (NCI-H209, SV-E, LD-T, and MO-A) derived from metastatic carcinomas, lack GJIC (16). Similar to previous findings in human lung cancer cells, these SCLC metastatic cell lines typically exhibit low GJIC because of decreased Cx expression. SK-LuCi-6 presents higher GJIC levels, suggesting different GJIC functions in different subtypes of lung cancers.

Furthermore, different isoforms of Cxs have been directly associated with tumor progression. Ectopic expression of $\mathrm{Cx} 43$ in $\mathrm{LH}_{7}$ cell lines derived from the highly metastatic human pulmonary giant cell carcinoma, indicates low levels of $\mathrm{Cx} 43$ enabling reestablishment of intracellular communication, causing more normal characterization (17). Notably, in the human NSCLC-ad cells lines, HCC827 and PC9, Cx26 expression triggered EMT (epithelial-mesenchymal transition) 
involving the PI3K/Akt signaling pathway (18). Rodent lung cancer models have also provided valuable information on $\mathrm{Cx}$ association with lung cancer metastasis. In mouse lung cancer tissue, altered expression of $\mathrm{mCx} 26, \mathrm{mCx} 32, \mathrm{mCx} 37$, $\mathrm{mCx} 40, \mathrm{mCx} 43$, and $\mathrm{mCx} 45 \mathrm{mRNA}$ is observed during lung tumorigenesis compared to normal lung tissue (19). These $\mathrm{mCx}$ proteins are known to be engaged in GJIC in lung cancer. Rat Cx26 mRNA expression was reduced in lung adenocarcinomas and induced in rats using $\mathrm{N}$-nitrosobis(2hydroxypropyl)amine, suggesting $\mathrm{rCx} 26$ involvement in tumor development (20).

Therefore, the functional roles of Cxs are largely influenced by mechanisms, both dependent and independent of gap junctions, type of lung cancer, and stage of lung cancer progression.

\section{PRIMARY LUNG CANCER AND CXS EXPRESSION}

\section{Broken Steady State of Cx43 During Lung Cancer Progression}

It is reported that $\mathrm{Cx} 43$ serves as an inhibitor of lung tumorigenesis in the early stage; however, a recent study revealed that $\mathrm{Cx} 43$ can also function as a tumor promoter (1). This controversy could be attributed from the fate of $\mathrm{Cx} 43$ at different stages of lung cancer: early stage, before metastasis to other tissue, and in the advanced stage of primary lung cancer. Cx43 mRNA and protein can be detected in tissues obtained from early stage human lung cancer, although the expression level is lower and is nucleus-localized compared with heathy lung tissue (1). Interesting, Cx43 mRNA and protein expression are gradually decreased in normal lung tissue adjacent to the tumor tissue and was closely correlated with the distance from the tumor tissue as $\mathrm{Cx} 43$ expression was lower in areas closer to the tumor site (21). These data suggest that cancer cells negatively affect $\mathrm{Cx} 43$ expression in the surrounding normal lung cells. Moreover, it also implies that primary lung cancer cells appear to "isolate themselves" by preventing the GJICs between lung cancer cells and normal lung cells.

However, the picture is quite different in advanced lung tumor. In the advanced stage of lung tumor, Zhao et al. found that $\mathrm{Cx} 43$ expression is positive in human NSCLC tissue in the advanced stage of lung cancer. This study suggests that $\mathrm{Cx} 43$ can be an important biomarker for the progression of NSCLC from lower grades with undetectable $\mathrm{Cx} 43$ levels, to higher grades during metastasis with poor prognosis (22). Furthermore, the other study indicates that $\mathrm{Cx} 43$ can be a prognostic factor to forecast advanced NSCLC, as high $\mathrm{Cx} 43$ is associated with a positive prognosis; in contrast, lower $\mathrm{Cx} 43$ is associated poor prognosis (23). Higher Cx43 in A549 cells lead to cisplatin (cisdiaminodichloroplatinum) chemotherapy resistance and reversed the EMT (18). Alternatively, Cx43 reverses resistance against EMT inhibition by cisplatin in A549/cisplatin cells, and lower Cx43 expression was observed in A549/cisplatin cells in comparison to A549 cells. These cells acquired an
EMT phenotype with morphological changes, such as spindlelike fibroblasts (21). In $\mathrm{mCx} 43$ heterozygous knockout mice $\mathrm{mCx} 43^{+/-}, \mathrm{mCx} 43$ loss was associated with lung cancer aggression and a higher incidence of NSCLC-ad induced by the known carcinogen 7,12-dimethylbenzanthracene (24). The difference in expression levels may explain how $\mathrm{Cx} 43$ can play opposed roles as a tumor inhibitor and enhancer depending upon the stages of lung cancer development.

There are ample evidences supporting inhibitory function of CX43 and GJIC in lung cancer cell lines and primary lung cancer growth. Using lung cancer cell line, an assay performed in an in vitro by Ruch et al. showed that forced $\mathrm{Cx} 43$ expression in a human cancer cell line restored GJIC and reduced cell growth and tumorigenicity (25). More recently, the same group reported an association between $\mathrm{Cx} 43$ and the neoplastic transformation of lung cancer stem cells (CSCs). Cx43 reversed some tumor features, and reduced the number of lung CSC in human (26). In the cell lines and tissue of lung tumor from mouse, GJIC is commonly defective, and the loss of GJ proteins, such as $\mathrm{mCx} 43$, results in the loss of a crucial component in intercellular communication and a vital intermediary of regulation in the phenotype and homeostasis (27). An interesting proteomic analysis assay was performed using the mouse lung tumorigenic cell line E9-2, to understand how GJIC regulates tumorigenesis. Altered levels of protein disulfide isomerase, gelsolin-like protein, $\alpha$-enolase, and aldolase $\mathrm{A}$, were observed upon abrogation of $\mathrm{mCx} 43$ by transfection (28). In mouse models, the loss of one $\mathrm{mCx} 43$ allele alone resulted in a higher incidence of lung lesions (29). However, a study demonstrated that $\mathrm{mCx} 43$ loses its tumor-suppressing function in advanced carcinogenesis, thus, $\mathrm{mCx} 43$ is considered as a conditional tumor suppressor (29). Mice with one deleted allele of the $\mathrm{mCx} 43$ gene $\left(\mathrm{mCx} 43^{+/-}\right), \mathrm{mCx} 43$ loss was associated with lung cancer aggression and a higher incidence of NSCLC-ad induced by the known carcinogen 7,12-dimethylbenzanthracene (24).

In the human NSCLC-ad A549 cell line, evidence supports that $\mathrm{Cx}$ is pro-tumorigenic particularly in cancer-associated fibroblasts, which accelerate the malignant progression of NSCLCs by forming Cx43-formed unidirectional GJIC from cancer-associated fibroblasts to A549 cells (30). The above-mentioned studies reveal a link between disruption of the steady state of Cx43 GJIC and lung tumorigenesis. This also indicates that maintaining the balance of the Cx43 steady state could be an important strategy for inhibition of lung tumorigenesis.

\section{Regulation of Cx43 During NSCLC Development}

It is important to consider how $\mathrm{Cx} 43$ is regulated during lung cancer development and tumorigenicity advancement and metastasis. However, there are very limited published studies concerning the regulatory mechanism of $\mathrm{Cx} 43$ during lung cancer development. Hypoxia activates the P53/MDM2 axis and induces $\mathrm{Cx} 43$ internalization (31). Cx43 is moved from the membrane to cytoplasm, where it is degraded (31), and the lower $\mathrm{Cx} 43$ levels promote EMT, inducing proliferation and tumorigenicity in human NSCLC tissue and cells (31). In contrast, p38 MAPK activation and JNK inhibition increases 
Cx43-mediated cell-cell communication via in human lung neoplastic cells and this activation is induced by 4-phenyl-3butenoic acid (32). Low Cx43 expression is also significantly associated with CpG island hypermethylation (CIH) in NSCLC. The level of $\mathrm{CIH}$ involved in poorly differentiated tumors and those caused by heavy smoking presented a weak $\mathrm{Cx} 43$ staining and ZO-1 or E-cadherin expression (33). Several studies indicated that $\mathrm{Cx} 43$ and E-cadherin are useful biomarkers in NSCLCs (22). Yeh and $\mathrm{Hu}$ proposed an explanation for conflicting reports on the functions of $\mathrm{Cx} 43$. They claimed that GJIC deficiency in the lung cancer A549 cells was mediated by oxidized beta-carotene, along with phosphorylation and abnormal positioning of $\mathrm{Cx} 43$ (34).

\section{Cx26, Cx31.1, Cx32, and Cx30.3 Functions in Lung Cancer Suppression}

In addition to $\mathrm{Cx} 43$, other $\mathrm{Cx}$ subtypes are also involved in lung cancer cell proliferation, EMT, tumorgenicity, and metastasis. In hypoxia induced human pulmonary epithelial cells, low levels of Cx26 promoted EMT, inducing proliferation and tumorigenicity of cancer cells (31). In the A549 NSCLC-ad cell line expressing Cx26, the PI3K/AKT signaling pathway is involved in EMT (18). In human NSCLC cell lines, Cx31.1 expression was reduced and inversely correlated with lung cancer metastasis. Notably, Cx31.1 promoted the expression of cytokeratin, a marker of epithelial cell, and inhibited the expression of vimentin, a marker of mesenchymal cells (35). These data indicate that Cx31.1 induced limited shift from a mesenchymal phenotype towards an epithelial one, and that Cx31.1 in NSCLC can be antitumorigenic. The interesting link between Cx31.1 degradation by autophagy and tumor suppression in NSCLC H1299 cells (36) should be further investigated.

mCx32-knockout (KO) mice displayed increased tissuespecific sensitivity to radiation-induced tumorigenesis in the lung. mCx32 suppresses mouse lung tumorigenesis by altering the activation of the MAPK pathway in a p27 status-dependent manner (37).

In contrast to other $\mathrm{Cxs}, \mathrm{mCx} 30.3$ promoted lung tumor growth and metastasis in a syngeneic mouse model, and its overexpression enhanced the sphere-forming ability and anchorage-independent growth of cancer cells (38). It is important to examine $\mathrm{Cx} 30.3$ in human tissue and cell lines to determine the utility of Cx30.3 as a diagnostic and prognostic biomarker for human lung cancer.

\section{ENDOTHELIAL CXS EXPRESSION AND IMPLICATION IN LUNG CANCER CELL EXTRAVASATION}

\section{Endothelial Cxs Promote Lung Cancer Cell Diapedesis}

Cxs are expressed in endothelial cells. GJs regulate endothelial cell stiffness, a crucial physical characteristic related to several vascular pathologies. Tumor necrosis factor- $\alpha$ (TNF $\alpha)$ temporarily increases the stiffness of endothelial cells and is manipulated by the interaction of cells and rearrangement of the cytoskeleton (39). Additionally, an important Cx43 mediated signaling pathway (EGF-ERK1/2-FAK-RhoA-Rac1) was shown to determine the efficiency of A549 cell diapedesis. A549 cellinduced activation of human umbilical vein endothelial cells correlated with an increased abundance of $\mathrm{Cx} 43$ plaques on a co-culture of both cell types. Furthermore, loss of Cx43-GJIC in treatment using 18 - $\alpha$-glycyrrhetinic acid and siRNA caused a weakened activation of endothelial cells cell in the human umbilical vein (40). Pannexin1, which only forms hemichannels, is functionally notable; the permeability of vascular endothelial cells is regulated by Pannexin1 (41). Additionally, in retinal vascular endothelial cells, non-junctional Cx43 decreased the permeability of monolayer cells, and inhibited apoptosis mediated by high glucose (42). Cx-mediated endothelial cell dysfunction is essential for lung cancer metastasis, and endothelial cell dysfunction disrupts the cell integrity by causing inflammation. Consequently, integrity and permeability are to be protected by $\mathrm{Cx}$ in the endothelial cells. Aspirin alleviates endothelial cell dysfunction by inhibiting activation of the NLRP3 inflammasome in lipopolysaccharideinduced vascular injury (43).

Additionally, the human endothelial cell line, EAhy 926, treated with IL-1 $\beta / \mathrm{TNF} \alpha$ and high glucose, in $\mathrm{Cx} 43$ hemichannel inhibited and reduced ATP release (44). Further, homeostasis and intracellular communication were regulated by $\mathrm{Cx} 37$ and $\mathrm{Cx} 40$ by interaction with NOS (nitric oxide synthase) in the endothelial cells $(45,46)$. Importantly, compared to non-tumor lung samples, the higher NOS was found in the NSCLC-ad (47). However, effective strategies for identifying functional GJs between lung cancer cells and endothelial Cxs during the intravasation of lung cancer cells into blood vessels are limited.

\section{Cx43 in the Brain Endothelial Barrier Interacts With Lung Cancer Cells}

Cx43 GJs are associated with hyperpermeability in the brain endothelial barrier. $\mathrm{Cx} 43$ is incorporated into the blood-brain barrier (BBB) junction complex, and the aberrantly increased Cx43 GJs regulate the permeability in a tight junctiondependent manner in the brain endothelial barrier (48). Additionally, $\mathrm{Cx} 43$ regulates the homeostasis of ions, $\mathrm{pH}$, and permeability in the BBB. In a previous review, supporting evidence was described regarding the role of astroglial cells and Cxs in manipulating the permeability of $\mathrm{BBB}$, initiated by infectious pathogenesis (49). HIV-infected astrocytes disrupt BBB integrity via a gap-junction-dependent mechanism due to endothelial apoptosis (50). cAMP activates cyclic nucleotide-gated channels, thereby inducing $\mathrm{Ca}^{2+}$ influx, leading to increased GJ coupling. Cyclic nucleotidegated channels act as a physiological link that integrates GJ coupling into adenosine receptor-dependent signaling of $\mathrm{BBB}$ endothelial cells (51). Opening of the Cx43 hemichannel, polarized by acute ischemic stroke-mimicking conditions disrupted the transport function of $\mathrm{BBB}$, and intracellular 
taurine and ATP were released in the BBB endothelial cells rat (TR-BBB13 cells) and human (hCMEC/D3 cells) origins (52). However, few studies have demonstrated GJ communication between lung cancer cells and endothelial Cxs during the extravasation of lung cancer cells and their crossing into the BBB.

During extravasation, endothelial cell $\mathrm{Cx} 37, \mathrm{Cx} 40$, and $\mathrm{Cx} 43$ contribute to lung cancer cell diapedesis from vessels. Additionally, brain endothelial Cx43 helps lung tumor cells to traverse to the brain (Figure 2). Thus, we concentrated on the function of GJs interaction between lung tumor and the endothelium, as well as their effects on lung cancer brain metastasis.

\section{ASTROCYTE CXS ARE INVOLVED IN BRAIN METASTASIS OF LUNG CANCER}

\section{Astrocyte Cxs Contribute to Lung Cancer Brain Metastasis}

The interplay of the Cx43 junction channel with astrocytes and lung tumors promotes metastasis. Lung cancer cells require a growth-permissive microenvironment to survive, which is provided by the astrocytes (14). However, astrocytes can kill most infiltrated lung cancer cells (53) and thus, are emerging as essential regulators of brain metastasis (54). Brain metastatic cancer cells could form gap-junctional networks with the astrocytes and transfer cGMP to astrocytes, leading to the activation of the STING pathway, resulting in the production of interferon- $\alpha$ and TNF (55). Additionally, activating the MAPK/ERK signaling pathway promotes brain metastasis of lung cancer cells via a microRNA-330-3p-mediated mechanism (56). Subsequently, tumor cells and astrocytes are mutually stimulated in the microenvironment of brain metastasis by specific inflammatory cytokines, and this mutual relationship promotes lung cancer metastasis and its development in the brain (57). Brain-metastasized lung cancers show increased expression of nuclear beta-catenin, which increases Cx43 expression (58). Similarly, overexpression of Cx30.3 has been found to increase lung cancer metastasis (38). Furthermore, suppressors of GJs between lung A549 CSCs and astrocytes, such as AS602801, are an anti-CSC drug candidate to suppress brain metastasis (59).

\section{Astrocyte Cx43 Hemichannels Are Involved in Lung Cancer Brain Metastasis}

Cx43 hemichannels, as well as GJIC in the astrocytes, may be involved in lung cancer brain metastasis. Astrocytes express high levels of $\mathrm{Cx} 43$, and the inhibition of cytokine-induced $\mathrm{Cx} 43$ hemichannels in astrocytes has a neuroprotective effect (60). Additionally, astrocyte $\mathrm{Cx} 43$ hemichannels increase the release of dickkopf-1 protein during HIV infection, thus contributing to brain pathogenesis observed in HIV-infected individuals (61). Notably, the activity of GJIC and hemichannel were differentially blocked by general anesthetics (propofol, ketamine, and dexmedetomidine), and had similar effects on neuronal hemichannels (62). In Parkinson's disease, astrocytes Cx43 hemichannel activity regulated midbrain dopamine neuron degeneration in a glucocorticoid receptor-dependent manner. Increased $\mathrm{Cx} 43$ hemichannel activity was found in vivo in MPTP-intoxicated mice, and decreasing its activity by use of the hemichannel blocker TAT-Gap 19 peptide, increased dopamine neuron and microglial activation (63). Studies of osteocytes have shown that Cx43 hemichannels suppressed breast cancer growth and bone metastasis (12). Proinflammatory cytokines reduced $\mathrm{Cx} 43$ levels on the cell surface in activated microglia (64). Notably, NSCLC development was enhanced by circ_ZNF124, which was targeted by miR-337-3p directly to downregulate the JAK2/STAT3 signaling pathway (65), thereby providing indirect evidence of the role of the GJastroglial-STAT3 axis in lung cancer brain metastasis.

\section{Factors Affecting Astrocyte Cx43 Expression Are Associated With Lung Cancer Metastasis}

Certain types of stress can affect $\mathrm{Cx} 43$ expression in astrocytes, and may be associated with lung cancer metastasis; however, direct evidence of this association is insufficient. Astrocytes Cx43 GJs ultrastructure changed in an oxygen-glucose

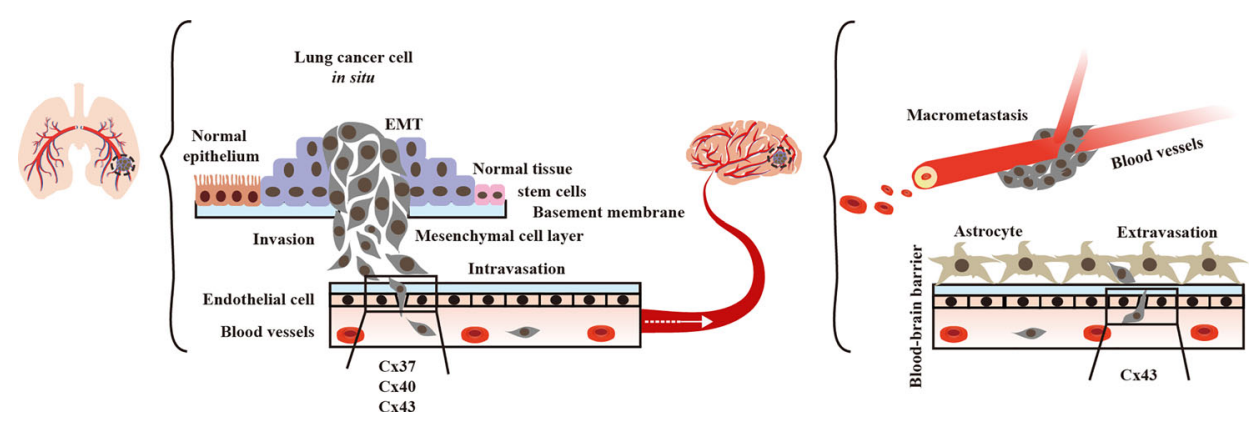

FIGURE 2 | Endothelial Connexins (Cxs) allow lung cancer diapedesis. In blood vessels, endothelial Cx37, Cx40, and Cx43 contribute to the of lung tumor cells (40, $45,46)$. 
A

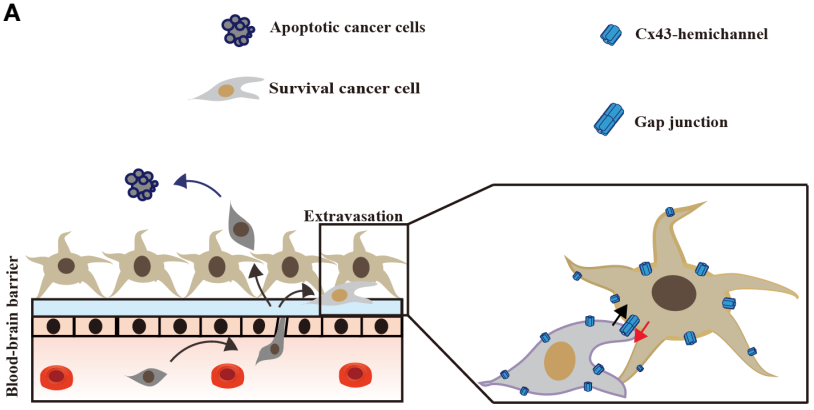
$\therefore$ Anti-tumor drug $\because$ Chemotherapy



FIGURE 3 | Astrocyte Cx43 interaction with brain metastases lung tumor. (A) At the occurrence of lung cancer brain metastasis, astrocytes kill most lung cancer cells that cross the blood-brain-barrier; few lung cancer cells survive form Cx43 gap junctions with astrocytes. (B) In the late stage of brain metastasis, Cx43 gap junctions between astrocytes and surviving lung cancer cells confer resistance against chemotherapy $(53,58)$.

deprivation mouse model. Oxygen-glucose deprivationCx43 metastasis-associated lung adenocarcinoma transcript I (MALAT1) may be related to lung cancer brain metastasis (66). Notably, MALAT1, the long noncoding RNA, protected human brain microvascular endothelial cells by inhibiting apoptosis induced by oxygen-glucose deprivation and reoxygenation (67). Norepinephrine is one of the most potent stimulators of tumor cell migration, and drives metastatic development of PC3 human prostate cancer (68). It may be interesting to study the norepinephrine-Cx43-lung cancer brain metastasis axis. Notably, junctional channels between lung cancer cells and astrocytes induce resistance to chemotherapy (69). PC-14 NSCLC brain metastases were protected from chemotherapy by astrocytes via a mechanism of endothelin-dependent signaling (70).

In the brain, $\mathrm{Cx} 43$ in astrocytes inhibits invading lung cancer cells at an early stage, and forms GJs with surviving lung cancer cells. In the late stage, Cx43 GJ channels between lung cancer cells and astrocytes promote chemotherapy resistance (Figure 3).

\section{CONCLUDING REMARKS}

A deeper understanding of the underlying mechanisms of $\mathrm{Cx}$ mediated brain metastasis, and regrowth of lung tumors will help in improving strategies for prevention of lung cancer metastasis. Following primary tumor removal, the goal of systemic therapy should be to prevent relapse. However, adjuvant therapy agents target growing lung cancer cells rather than inhibiting metastasis. Cxs can function as both lung cancer suppressors and promoters depending on the isoforms, stages, and the type of lung cancer. Furthermore, endothelial Cxs contribute to lung cancer diapedesis and astrocyte Cxs contribute to the brain metastasis of lung cancer cells. Thus, using endothelial Cxs to build barriers against lung cancer diapedesis may be the best strategy for inhibiting the metastasis of lung cancer to the brain. There is a lack of research models and an appropriate lung cancer type to study all Cx isoforms, as well as tracking the entire process of lung cancer-brain metastasis. Furthermore, it is important to study lung cancer metastasis in the environmental context with potential lung carcinogens, such as coal combustion emissions. The findings of such investigations on the $\mathrm{Cx}$ isoforms and lung cancer types should be compared to global geographic variations, as reflected by the lung cancer rate in Xuanwei, China, which is the highest in the world $(71,72)$. Extrapolating knowledge from studies on Cxs from other cancer types, to lung cancer and brain metastasis may also foster the development of more effective therapeutic approaches.

There is a lack of therapeutics for lung cancers, but mimics peptides of Cxs currently under clinical trials for other diseases (73) and could be used to cancer therapeutics.

\section{AUTHOR CONTRIBUTIONS}

$\mathrm{K}-\mathrm{JL}, \mathrm{Y}-\mathrm{CH}$, and JJ designed and wrote the manuscript. C-XC constructed figures. J-PY collected references. EC proofread the manuscript. All authors contributed to the article and approved the submitted version.

\section{FUNDING}

K-JL was supported by the Science and Technology Planning Project in Key Areas of Yunnan Province (202001BB050002) and the NSFC (31471823, 31772225, 31260448, 31060251) and has been awarded a State Scholarship Fund to pursue studies in the United States of America as a visiting scholar. Y-CH was supported by the NSFC (81960335), Key Project of International Cooperation of Science and Technology Innovation between Governments, and National Key Research and Development Plan of China (2016YEE0103400). JJ was supported by the Welch Foundation (AQ-1507) and NIH (CA196214). 


\section{REFERENCES}

1. Aasen T, Sansano I, Montero MA, Romagosa C, Temprana-Salvador J, MartinezMarti A, et al. Insight into the Role and Regulation of Gap Junction Genes in Lung Cancer and Identification of Nuclear $\mathrm{Cx} 43$ as a Putative Biomarker of Poor Prognosis. Cancers (Basel) (2019) 11:1-20. doi: 10.20944/preprints201902.0109.v1

2. Ito A, Koma Y, Uchino K, Okada T, Ohbayashi C, Tsubota N, et al. Increased expression of connexin 26 in the invasive component of lung squamous cell carcinoma: significant correlation with poor prognosis. Cancer Lett (2006) 234:239-48. doi: 10.1016/j.canlet.2005.03.049

3. Rackauskas M, Neverauskas V, Skeberdis VA. Diversity and properties of connexin gap junction channels. Medicina (Kaunas) (2010) 46:1-12. doi: 10.3390/medicina46010001

4. Evans WH, Martin PE. Gap junctions: structure and function (Review). Mol Membr Biol (2002) 19:121-36. doi: 10.1080/09687680210139839

5. Willecke K, Eiberger J, Degen J, Eckardt D, Romualdi A, Guldenagel M, et al. Structural and functional diversity of connexin genes in the mouse and human genome. Biol Chem (2002) 383:725-37. doi: 10.1515/BC.2002.076

6. Sohl G, Willecke K. Gap junctions and the connexin protein family. Cardiovasc Res (2004) 62:228-32. doi: 10.1016/j.cardiores.2003.11.013

7. Ravera S, Bartolucci M, Adriano E, Garbati P, Ferrando S, Ramoino P, et al. Support of Nerve Conduction by Respiring Myelin Sheath: Role of Connexons. Mol Neurobiol (2016) 53:2468-79. doi: 10.1007/s12035-015-9216-0

8. Cheung G, Chever O, Rouach N. Connexons and pannexons: newcomers in neurophysiology. Front Cell Neurosci (2014) 8:348. doi: 10.3389/fncel.2014.00348

9. Siebert AP, Ma ZM, Grevet JD, Demuro A, Parker I, Foskett JK. Structural and functional similarities of calcium homeostasis modulator 1 (CALHM1) ion channel with connexins, pannexins, and innexins. J Biol Chem (2013) 288:6140-53. doi: 10.1074/jbc.M112.409789

10. Aasen T, Mesnil M, Naus CC, Lampe PD, Laird DW. Gap junctions and cancer: communicating for 50 years. Nat Rev Cancer (2016) 16:775-88. doi: 10.1038/nrc.2016.105

11. Zhou JZ, Jiang JX. Gap junction and hemichannel-independent actions of connexins on cell and tissue functions-an update. FEBS Lett (2014) 588:118692. doi: 10.1016/j.febslet.2014.01.001

12. Zhou JZ, Riquelme MA, Gu S, Kar R, Gao X, Sun L, et al. Osteocytic connexin hemichannels suppress breast cancer growth and bone metastasis. Oncogene (2016) 35:5597-607. doi: 10.1038/onc.2016.101

13. Massague J, Obenauf AC. Metastatic colonization by circulating tumour cells. Nature (2016) 529:298-306. doi: 10.1038/nature17038

14. Obenauf AC, Massagué J. Surviving at a distance: organ specific metastasis. Trends Cancer (2015) 1:76-91. doi: 10.1016/j.trecan.2015.07.009

15. Travis WD, Brambilla E, Nicholson AG, Yatabe Y, Austin JHM, Beasley MB, et al. The 2015 World Health Organization Classification of Lung Tumors: Impact of Genetic, Clinical and Radiologic Advances Since the 2004 Classification. J Thorac Oncol (2015) 10:1243-60. doi: 10.1097/JTO.0000000000000630

16. Tomai E, Brownell HL, Tufescu TV, Reid K, Campling BG, Raptis L. Gap junctional communication in cultured human lung carcinoma cells. Lung Cancer (1999) 23:223-31. doi: 10.1016/S0169-5002(99)00016-1

17. Xu HT, Li QC, Zhang YX, Zhao Y, Liu Y, Yang ZQ, et al. Connexin 43 recruits Ecadherin expression and inhibits the malignant behaviour of lung cancer cells. Folia Histochem Cytobiol (2008) 46:315-21. doi: 10.2478/v10042-008-0057-9

18. Yang J, Qin G, Luo M, Chen J, Zhang Q, Li L, et al. Reciprocal positive regulation between Cx26 and PI3K/Akt pathway confers acquired gefitinib resistance in NSCLC cells via GJIC-independent induction of EMT. Cell Death Dis (2015) 6:e1829-9. doi: 10.1038/cddis.2015.197

19. Udaka N, Miyagi Y, Ito T. Connexin expression in mouse lung tumor. Cancer Lett (2007) 246:224-9. doi: 10.1016/j.canlet.2006.02.020

20. Shimizu K, Shimoichi Y, Hinotsume D, Itsuzaki Y, Fujii H, Honoki K, et al. Reduced expression of the Connexin26 gene and its aberrant DNA methylation in rat lung adenocarcinomas induced by $\mathrm{N}$-nitrosobis(2hydroxypropyl)amine. Mol Carcinog (2006) 45:710-4. doi: 10.1002/mc.20207

21. Yu M, Zhang C, Li L, Dong S, Zhang N, Tong X. Cx43 reverses the resistance of A549 lung adenocarcinoma cells to cisplatin by inhibiting EMT. Oncol Rep (2014) 31:2751-8. doi: 10.3892/or.2014.3163

22. Zhao JQ, Sun FJ, Liu SS, Yang J, Wu YQ, Li GS, et al. Expression of connexin 43 and E-cadherin protein and mRNA in non-small cell lung cancers in
Chinese patients. Asian Pac J Cancer Prev (2013) 14:639-43. doi: 10.7314/ APJCP.2013.14.2.639

23. Du G, Yang Y, Zhang Y, Sun T, Liu W, Wang Y, et al. Thrombocytosis and immunohistochemical expression of connexin 43 at diagnosis predict survival in advanced non-small-cell lung cancer treated with cisplatin-based chemotherapy. Cancer Chemother Pharmacol (2013) 71:893-904. doi: 10.1007/s00280-013-2080-6

24. de Oliveira KD, Tedardi MV, Cogliati B, Dagli ML. Higher incidence of lung adenocarcinomas induced by DMBA in connexin 43 heterozygous knockout mice. BioMed Res Int (2013) 2013:618475. doi: 10.1155/2013/618475

25. Ruch RJ, Cesen-Cummings K, Malkinson AM. Role of gap junctions in lung neoplasia. Exp Lung Res (1998) 24:523-39. doi: 10.3109/01902149809087384

26. Ruch RJ. Connexin 43 Suppresses Lung Cancer Stem Cells. Cancers (Basel) (2019) 11:1-17. doi: 10.3390/cancers11020175

27. Ruch RJ, Porter S, Koffler LD, Dwyer-Nield LD, Malkinson AM. Defective gap junctional intercellular communication in lung cancer: loss of an important mediator of tissue homeostasis and phenotypic regulation. Exp Lung Res (2001) 27:231-43. doi: 10.1080/019021401300053984

28. Peebles KA, Duncan MW, Ruch RJ, Malkinson AM. Proteomic analysis of a neoplastic mouse lung epithelial cell line whose tumorigenicity has been abrogated by transfection with the gap junction structural gene for connexin 43, Gjal. Carcinogenesis (2003) 24:651-7. doi: 10.1093/carcin/bgg008

29. Fukumasu H, Avanzo JL, Sanches DS, Mennecier G, Mori CM, Dagli ML. Higher susceptibility of spontaneous and NNK-induced lung neoplasms in connexin 43 deficient CD1 x AJ F1 mice: paradoxical expression of connexin 43 during lung carcinogenesis. Mol Carcinog (2013) 52:497-506. doi: 10.1002/mc.21884

30. Luo M, Luo Y, Mao N, Huang G, Teng C, Wang H, et al. Cancer-Associated Fibroblasts Accelerate Malignant Progression of Non-Small Cell Lung Cancer via Connexin 43-Formed Unidirectional Gap Junctional Intercellular Communication. Cell Physiol Biochem (2018) 51:315-36. doi: 10.1159/000495232

31. Zeng SG, Lin X, Liu JC, Zhou J. Hypoxiainduced internalization of connexin 26 and connexin 43 in pulmonary epithelial cells is involved in the occurrence of nonsmall cell lung cancer via the P53/MDM2 signaling pathway. Int J Oncol (2019) 55:845-59. doi: 10.3892/ijo.2019.4906

32. Matesic DF, Sidorova TS, Burns TJ, Bell AM, Tran PL, Ruch RJ, et al. p38 MAPK activation, JNK inhibition, neoplastic growth inhibition, and increased gap junction communication in human lung carcinoma and Ras-transformed cells by 4-phenyl3-butenoic acid. J Cell Biochem (2012) 113:269-81. doi: 10.1002/jcb.23353

33. Jinn Y, Inase N. Connexin 43, E-cadherin, beta-catenin and ZO-1 expression, and aberrant methylation of the connexin 43 gene in NSCLC. Anticancer Res (2010) 30:2271-8.

34. Yeh S-L, Hu M-L. Oxidized $\beta$-carotene inhibits gap junction intercellular communication in the human lung adenocarcinoma cell line A549. Food Chem Toxicol (2003) 41:1677-84. doi: 10.1016/S0278-6915(03)00192-3

35. Zhang D, Chen C, Li Y, Fu X, Xie Y, Li Y, et al. Cx31.1 acts as a tumour suppressor in non-small cell lung cancer (NSCLC) cell lines through inhibition of cell proliferation and metastasis. J Cell Mol Med (2012) 16:1047-59. doi: 10.1111/j.1582-4934.2011.01389.x

36. Zhu X, Ruan Z, Yang X, Chu K, Wu H, Li Y, et al. Connexin 31.1 degradation requires the Clathrin-mediated autophagy in NSCLC cell H1299. J Cell Mol Med (2015) 19:257-64. doi: 10.1111/jcmm.12470

37. King TJ, Gurley KE, Prunty J, Shin JL, Kemp CJ, Lampe PD. Deficiency in the gap junction protein connexin32 alters p27Kip1 tumor suppression and MAPK activation in a tissue-specific manner. Oncogene (2005) 24:1718-26. doi: 10.1038/sj.onc. 1208355

38. Lin YP, Wu JI, Tseng CW, Chen HJ, Wang LH. Gjb4 serves as a novel biomarker for lung cancer and promotes metastasis and chemoresistance via Src activation. Oncogene (2019) 38:822-37. doi: 10.1038/s41388-018-0471-1

39. Okamoto T, Kawamoto E, Takagi Y, Akita N, Hayashi T, Park EJ, et al. Gap junction-mediated regulation of endothelial cellular stiffness. Sci Rep (2017) 7:6134. doi: 10.1038/s41598-017-06463-x

40. Piwowarczyk K, Kwiecien E, Sosniak J, Zimolag E, Guzik E, Sroka J, et al. Fenofibrate Interferes with the Diapedesis of Lung Adenocarcinoma Cells through the Interference with Cx43/EGF-Dependent Intercellular Signaling. Cancers (Basel) (2018) 10:1-17. doi: 10.3390/cancers10100363

41. Sharma AK, Charles EJ, Zhao Y, Narahari AK, Baderdinni PK, Good ME, et al. Pannexin-1 channels on endothelial cells mediate vascular inflammation 
during lung ischemia-reperfusion injury. Am J Physiol Lung Cell Mol Physiol (2018) 315:L301-12. doi: 10.1152/ajplung.00004.2018

42. Kim D, Mouritzen U, Larsen BD, Roy S. Inhibition of Cx43 gap junction uncoupling prevents high glucose-induced apoptosis and reduces excess cell monolayer permeability in retinal vascular endothelial cells. Exp Eye Res (2018) 173:85-90. doi: 10.1016/j.exer.2018.05.003

43. Zhou X, Wu YJ, Ye LF, Wang YT, Zhang KM, Wang LJ, et al. Aspirin alleviates endothelial gap junction dysfunction through inhibition of NLRP3 inflammasome activation in LPS-induced vascular injury. Acta Pharm Sin B (2019) 9:711-23. doi: 10.1016/j.apsb.2019.02.008

44. Saez JC, Contreras-Duarte S, Gomez GI, Labra VC, Santibanez CA, GajardoGomez R, et al. Connexin 43 Hemichannel Activity Promoted by ProInflammatory Cytokines and High Glucose Alters Endothelial Cell Function. Front Immunol (2018) 9:1899. doi: 10.3389/fimmu.2018.01899

45. Meens MJ, Alonso F, Le Gal L, Kwak BR, Haefliger JA. Endothelial Connexin37 and Connexin40 participate in basal but not agonist-induced NO release. Cell Commun Signal (2015) 13:34. doi: 10.1186/s12964-015-0110-1

46. Pfenniger A, Derouette JP, Verma V, Lin X, Foglia B, Coombs W, et al. Gap junction protein $\mathrm{Cx} 37$ interacts with endothelial nitric oxide synthase in endothelial cells. Arterioscler Thromb Vasc Biol (2010) 30:827-34. doi: 10.1161/ATVBAHA.109.200816

47. Fujimoto H, Ando Y, Yamashita T, Terazaki H, Tanaka Y, Sasaki J, et al. Nitric oxide synthase activity in human lung cancer. Jpn J Cancer Res (1997) 88:1190-8. doi: 10.1111/j.1349-7006.1997.tb00348.x

48. Johnson AM, Roach JP, Hu A, Stamatovic SM, Zochowski MR, Keep RF, et al. Connexin 43 gap junctions contribute to brain endothelial barrier hyperpermeability in familial cerebral cavernous malformations type III by modulating tight junction structure. FASEB J (2018) 32:2615-29. doi: 10.1096/ f. 201700699R

49. De Bock M, Leybaert L, Giaume C. Connexin Channels at the Glio-Vascular Interface: Gatekeepers of the Brain. Neurochem Res (2017) 42:2519-36. doi: 10.1007/s11064-017-2313-x

50. Eugenin EA, Clements JE, Zink MC, Berman JW. Human immunodeficiency virus infection of human astrocytes disrupts blood-brain barrier integrity by a gap junction-dependent mechanism. J Neurosci (2011) 31:9456-65. doi: 10.1523/JNEUROSCI.1460-11.2011

51. Bader A, Bintig W, Begandt D, Klett A, Siller IG, Gregor C, et al. Adenosine receptors regulate gap junction coupling of the human cerebral microvascular endothelial cells hCMEC/D3 by $\mathrm{Ca}(2+)$ influx through cyclic nucleotide-gated channels. J Physiol (2017) 595:2497517. doi: $10.1113 / \mathrm{JP} 273150$

52. Tachikawa M, Murakami K, Akaogi R, Akanuma SI, Terasaki T, Hosoya KI. Polarized hemichannel opening of pannexin 1 /connexin 43 contributes to dysregulation of transport function in blood-brain barrier endothelial cells. Neurochem Int (2020) 132:104600. doi: 10.1016/j.neuint.2019.104600

53. Valiente M, Obenauf AC, Jin X, Chen Q, Zhang XH, Lee DJ, et al. Serpins promote cancer cell survival and vascular co-option in brain metastasis. Cell (2014) 156:1002-16. doi: 10.1016/j.cell.2014.01.040

54. Ferraro GB, Kodack DP, Askoxylakis V, Jain RK. Closing the gap: astrocytes and brain metastasis. Cell Res (2016) 26:973-4. doi: 10.1038/cr.2016.96

55. Chen Q, Boire A, Jin X, Valiente M, Er EE, Lopez-Soto A, et al. Carcinomaastrocyte gap junctions promote brain metastasis by cGAMP transfer. Nature (2016) 533:493-8. doi: 10.1038/nature18268

56. Wei C-H, Wu G, Cai Q, Gao X-C, Tong F, Zhou R, et al. MicroRNA-330-3p promotes cell invasion and metastasis in non-small cell lung cancer through GRIA3 by activating MAPK/ERK signaling pathway. J Hematol Oncol (2017) 10:125. doi: 10.1186/s13045-017-0493-0

57. Seike T, Fujita K, Yamakawa Y, Kido MA, Takiguchi S, Teramoto N, et al. Interaction between lung cancer cells and astrocytes via specific inflammatory cytokines in the microenvironment of brain metastasis. Clin Exp Metastasis (2011) 28:13-25. doi: 10.1007/s10585-010-9354-8

58. Kafka A, Tomas D, Beroš V, Pécina HI, Zeljko M, Pećina-Šlaus N. Brain metastases from lung cancer show increased expression of DVL1, DVL3 and beta-catenin and down-regulation of E-cadherin. Int J Mol Sci (2014) 15:10635-51. doi: 10.3390/ijms150610635

59. Kuramoto K, Yamamoto M, Suzuki S, Sanomachi T, Togashi K, Seino S, et al. AS602801, an Anti-Cancer Stem Cell Drug Candidate, Suppresses Gap- junction Communication Between Lung Cancer Stem Cells and Astrocytes. Anticancer Res (2018) 38:5093-9. doi: 10.21873/anticanres.12829

60. Froger N, Orellana JA, Calvo CF, Amigou E, Kozoriz MG, Naus CC, et al. Inhibition of cytokine-induced connexin 43 hemichannel activity in astrocytes is neuroprotective. Mol Cell Neurosci (2010) 45:37-46. doi: 10.1016/j.mcn.2010. 05.007

61. Orellana JA, Saez JC, Bennett MV, Berman JW, Morgello S, Eugenin EA. HIV increases the release of dickkopf- 1 protein from human astrocytes by a $\mathrm{Cx} 43$ hemichannel-dependent mechanism. J Neurochem (2014) 128:752-63. doi: 10.1111/jnc. 12492

62. Liu XH, Gangoso E, Yi CJ, Jeanson T, Kandelman S, Mantz J, et al. General Anesthetics Have Differential Inhibitory Effects on Gap Junction Channels and Hemichannels in Astrocytes and Neurons. Glia (2016) 64:524-36. doi: 10.1002/glia.22946

63. Maatouk L, Yi C, Carrillo-de Sauvage MA, Compagnion AC, Hunot S, Ezan P, et al. Glucocorticoid receptor in astrocytes regulates midbrain dopamine neurodegeneration through connexin hemichannel activity. Cell Death Differ (2019) 26:580-96. doi: 10.1038/s41418-018-0150-3

64. Retamal MA, Froger N, Palacios-Prado N, Ezan P, Saez PJ, Saez JC, et al. Cx43 hemichannels and gap junction channels in astrocytes are regulated oppositely by proinflammatory cytokines released from activated microglia. J Neurosci (2007) 27:13781-92. doi: 10.1523/JNEUROSCI.2042-07.2007

65. Li Q, Huang Q, Cheng S, Wu S, Sang H, Hou J. Circ_ZNF124 promotes nonsmall cell lung cancer progression by abolishing miR-337-3p mediated downregulation of JAK2/STAT3 signaling pathway. Cancer Cell Int (2019) 19:291. doi: 10.1186/s12935-019-1011-y

66. Beckmann A, Grissmer A, Wolf S, Recktenwald J, Meier C. Oxygen-Glucose Deprivation in Mouse Astrocytes is Associated with Ultrastructural Changes in Connexin 43 Gap Junctions. Neuroscience (2019) 397:67-79. doi: 10.1016/ j.neuroscience.2018.11.043

67. Xin JW, Jiang YG. Long noncoding RNA MALAT1 inhibits apoptosis induced by oxygen-glucose deprivation and reoxygenation in human brain microvascular endothelial cells. Exp Ther Med (2017) 13:1225-34. doi: 10.3892/etm.2017.4095

68. Palm D, Lang K, Niggemann B, Drell T, Masur K, Zaenker KS, et al. The norepinephrine-driven metastasis development of PC-3 human prostate cancer cells in $\mathrm{BALB} / \mathrm{c}$ nude mice is inhibited by beta-blockers. Int J Cancer (2006) 118:2744-9. doi: 10.1002/ijc.21723

69. Menachem A, Makovski V, Bodner O, Pasmanik-Chor M, Stein R, Shomron N, et al. Intercellular transfer of small RNAs from astrocytes to lung tumor cells induces resistance to chemotherapy. Oncotarget (2016) 7:12489-504. doi: 10.18632 /oncotarget.7273

70. Lee HJ, Hanibuchi M, Kim SJ, Yu H, Kim MS, He J, et al. Treatment of experimental human breast cancer and lung cancer brain metastases in mice by macitentan, a dual antagonist of endothelin receptors, combined with paclitaxel. Neuro Oncol (2016) 18:486-96. doi: 10.1093/neuonc/now037

71. Chen Y, Li G, Lei Y, Yang K, Niu H, Zhao J, et al. Lung cancer family history and exposure to occupational/domestic coal combustion contribute to variations in clinicopathologic features and gene fusion patterns in non-small cell lung cancer. Thorac Cancer (2019) 10:695-707. doi: 10.1111/1759-7714.12987

72. Wong JYY, Downward GS, Hu W, Portengen L, Seow WJ, Silverman DT, et al. Lung cancer risk by geologic coal deposits: A case-control study of female never-smokers from Xuanwei and Fuyuan, China. Int J Cancer (2019) 144:2918-27. doi: 10.1002/ijc.32034

73. Delvaeye T, De Smet MAJ, Verwaerde S, Decrock E, Czekaj A, Vandenbroucke RE, et al. Blocking connexin 43 hemichannels protects mice against tumour necrosis factor-induced inflammatory shock. Sci Rep (2019) 9:16623. doi: 10.1038/s41598-019-52900-4

Conflict of Interest: The authors declare that the research was conducted in the absence of any commercial or financial relationships that could be construed as a potential conflict of interest.

Copyright ( 2020 Luo, Chen, Yang, Huang, Cardenas and Jiang. This is an open-access article distributed under the terms of the Creative Commons Attribution License (CC BY). The use, distribution or reproduction in other forums is permitted, provided the original author(s) and the copyright owner(s) are credited and that the original publication in this journal is cited, in accordance with accepted academic practice. No use, distribution or reproduction is permitted which does not comply with these terms. 